\title{
Diagnóstico prenatal citogenético: líquido amniótico versus vellosidades coriónicas
}

\author{
Elizabeth Silva Ospina*; Alejandro Giraldo Ríos**; Antonio José Bermúdez***
}

\section{RESUMEN}

OBJETIVO: Se presentan los resultados comparativos de un estudio prenatal citogenético en muestras de líquido amniótico (LA) y vellosidades coriónicas (VC).

PACIENTES Y METODOS: Se evaluaron 47 LA obtenidos durante el segundo trimestre de gestación y 25 VC del primer trimestre, de pacientes remitidas al Instituto Nacional de Salud cuyas gestaciones se encontraban con riesgo incrementado de presentar anomalías cromosómicas. Con las muestras de LA se realizaron cultivos largos combinando los métodos en frasco y en slide flask. Con las VC se utilizó el método de cultivo corto durante 24 y 48 horas. Se realizaron las bandas cromosómicas GTG y QFQ.

RESULTADOS: De las muestras tomadas adecuadamente se obtuvieron los resultados citogenéticos en el $97.6 \%$ de los LA y en el 95.6\% de las VC. EI 4.7\% de las muestras de LA y el 9\% de las muestras de VC presentaban alteraciones cromosómicas correspondientes a la constitución real del feto. Hubo un falso positivo en cada una de las muestras de LA (1.1\%) y VC. (4.5\%). Las alteraciones encontradas fueron tanto numéricas como estructurales: se encontraron dos trisomías 21, dos trisomías 18, una translocación balanceada entre los cromosomas 14 y 21 y un cromosoma $Y$ con ausencia del segmento fluorescente.

CONCLUSIONES: El éxito citogenético obtenido fue alto tanto para las muestras de LA como para las de VC. EI tipo de alteraciones cromosómicas encontradas fue el esperado para la población estudiada pero la frecuencia fue más alta que la reportada en la literatura.

PALABRAS CLAVES: Diagnóstico prenatal, citogenética, anomalías cromosómicas, líquido amniótico, vellosidades coriónicas.

\section{SUMMARY}

OBJECTIVE: A comparative analysis between amniotic fluid (AF) and chorion villous sampling (CVS) cytogenetics is presented.

PATIENTS AND METHODS: Forty seven second trimester AF samples and twenty five CVS were obtained from patients referred to the Colombian National Institute of Health, for incremented risk to chromosomal aberrations. The AF was long-term cultured in slide flask and, for the CVS, short-term (24 and 48 hours) cultures were performed. For both types of cultures, $\mathrm{G}$ and $\mathrm{Q}$ banding were analysed.

RESULTS: Cytogenetic results were observed in 97.6\% of AF samples and $95.6 \%$ of $\mathrm{CV}$, out of the samples taken in good condicions. In $4.7 \%$ of the AF samples and $9 \%$ of CVS, chromosomes abnormalities were observed indicating the real fetal chromosomal constitution. A false positive was found, in both AF (1.1\%) and CVS (4.5\%) cultures. The cytogenetic abnormalities observed were numerical and structural: trisomy 21 two, trisomy 18 two, Robertsonian translocation between the 14 and the 21 chromosome one, and one Y chromosome with an absent of the fluorescent segment.

CONCLUTIONS: The chromosome analysis was sucecessful in both types of samples, AF and CVS. The type of cytogenetic abnormalities found were the expected for the studied population, but the frequency was higher than that reported in the literature.

KEY WORDS: Prenatal diagnosis, cytogenetic, chromosome abnormalities, amniotic fluid, chorionic villi.

\footnotetext{
Microbióloga, Magister en Microbiología, Area Genética.

Médico Genetista y Magister en Salud Pública.

*** Médico, Magister en Bioquímica.
}

El tema fue presentado el 19 de septiembre de 1996 durante el II Congreso Colombiano de Genética realizado en Santafé de Bogotá. 


\section{Introducción}

El análisis cromosómico de las células fetales se inició en 1966 al demostrarse que en el líquido amniótico (LA) se encontraban células fetales viables, capaces de llevar a cabo mitosis en cultivos (1). Desde entonces se han mejorado los procedimientos de amniocentesis y de cultivos y se han desarrollado nuevos métodos para detectar prenatalmente diferentes trastornos, tanto cromosómicos como bioquímicos. A mediados de los años 80 fue posible realizar estudios cromosómicos en muestras de vellosidades coriónicas (VC) obtenidas por medio de sondas transcervicales guiadas ecográficamente. El fundamento para estudiar estas muestras se basa en el hecho de que genéticamente la placenta es considerada un órgano fetal, por consiguiente el estudio de las VC refleja indirectamente la constitución genética del feto.

El diagnóstico citogenético en LA y VC, se ofrece en los países desarrollados a las gestantes con un riesgo aumentado de presentar un feto con alguna anormalidad cromosómica. Estos riesgos se presentan en madres mayores de 35 años, o en quienes han tenido previamente un hijo con esta patología, o cuando uno de los padres es portador de una anomalía cromosómica balanceada (2). De la misma manera se estudian gestaciones con riesgo de enfermedades ligadas al cromosoma $X$ y también cuando en ecografías de rutina se detectan malformaciones del feto, acumulación del fluido nucal en el primer trimestre, retraso en el crecimiento intrauterino o desórdenes del LA. (3 y 4 )

Más recientemente se vienen estudiando desde el punto de vista citogenético, las gestaciones que presentan niveles anormales en el screening prenatal en suero materno. En la actualidad se recomienda realizarlo utilizando 4 marcadores: alfa-fetoproteína, gonadotrofina coriónica, estriol no conjugado e inhibina A (5).

Además, con células obtenidas por amniocentesis o por biopsia de corion actualmente es posible el diagnóstico molecular de un grupo cada vez mayor de enfermedades genéticas.

Se estima que por lo menos el $20 \%$ de las concepciones humanas portan alguna anomalía cromosómica, sin embargo la mayoría de estas se abortan espontáneamente (6). Se ha calculado que el $0.92 \%$ de los recién nacidos vivos presentan defectos cromosómicos numéricos o estructurales (7), de estos el más frecuente es el síndrome de Down, cuya prevalencia al nacimiento es de 1:800 recién nacidos vivos (8), cifra que es sensiblemente igual en todas las poblaciones del mundo.

A partir de los datos obtenidos con estudios de amniocentesis, se estima que la frecuencia de aberraciones cromosómicas en los fetos de madres mayores de 35 años es de 2,26\% y en el grupo por encima de 40 años el rango se eleva entre el $2 \%$ y el $5 \%$ (9). En Bogotá, de acuerdo con los datos del Servicio de Salud del Distrito, se calcula que el $0,3 \%$ de los nacimientos al año se producen en madres de más de 40 años y el 6,7\% corresponden a madres de más de 35 años. Si se estima que para
Bogotá el número de nacimientos al año es de 100.000 aproximadamente, alrededor de 300 a 6.700 nacimientos se presentarían respectivamente en estos grupos de edad.

Por otra parte, en esta misma ciudad se ha observado que la edad media de maternidad está incrementándose, sobre todo en personas con mayor nivel educativo y mayores ingresos económicos, por lo tanto la tendencia va hacia el aumento de embarazos en madres mayores de 35 años.

\section{Pacientes y métodos}

El grupo de pacientes seleccionados estuvo constituido por 112 gestantes remitidas al Instituto Nacional de Salud por diversos establecimientos del país, con indicaciones preconcepcionales, obstétricas o fetales, para la realización del diagnóstico prenatal citogenético. Antes de que se realizara la toma de la muestra cada una de las pacientes recibió asesoramiento genético y las que consultaron antes de la semana 12 de gestación eligieron libremente el procedimiento utilizado.

\section{Método de toma de muestra}

Las muestras fueron tomadas por obstetras que utilizaron la vía transabdominal (TA) para la extracción del LA y las vías TA o trans-cervical (TC) en la biopsia de VC. Todas las muestras fueron obtenidas con control ecográfico y en estrictas condiciones de asepsia.

\section{Método de estudio citogenético para LA}

Los LA se transportaron al laboratorio en las mismas jeringas en que fueron extraídos o en tubos estériles tapa rosca, a temperatura ambiente y protegidos de la luz.

Para realizar el análisis citogenético se siguió el protocolo desarrollado por el laboratorio de diagnóstico prenatal de la ciudad de Nueva York (PDL) (10), que consiste en que para cada muestra se realizan 3 siembras independientes, en este estudio las hicimos combinando los métodos in situ y en frasco. Una vez terminado el tiempo de cultivo se cosechan 2 de los 3 frascos y de cada uno de ellos se analizan 10 células ( 20 en total) bandeadas previamente, en este estudio se utilizaron de rutina las bandas GTG. En los casos en los que se encontraron discordancias o mosaicos se cosechó el tercer frasco y se incrementó a 50 el número de células estudiadas para definir el diagnóstico citogenético.

\section{Método de estudio citogenético para VC}

Cada muestra de vellosidades coriónicas fue extraída con una jeringa que contenía solución salina, heparina sódica, penicilina y estreptomicina. Posteriormente fue transferida asépticamente a una caja de Petrí, en la que se procedió con la ayuda de un estereoscopio, a identificar, limpiar y definir la cantidad de vellosidades extraídas. La muestra se consideró adecuada si presentaba 
vellosidades vitales y de peso aproximado de $10 \mathrm{mgs}$ como mínimo.

Se realizaron cultivos cortos de 24 horas (11) y 48 horas (12). El procedimiento de cosecha para ambos tipos de cultivo se hizo según el método descrito por Simoni y colaboradores (13), con algunas modificaciones, las cuales consistieron en una fijación previa al goteo utilizando una mezcla de metanol en alta concentración con ácido acético al 50\%, y colocando la suspensión suavemente sobre la lámina precalentada (14). Se realizaron bandas GTG y QFQ. Se estudiaron entre 10 y 15 metafases por muestra. En los casos en los cuales no fue posible el análisis adecuado de los cromosomas de la muestra de VC, se estableció la realización del estudio cromosómico por amniocentesis.

\section{Resultados}

De las 112 gestantes estudiadas, a 87 se les practicó la amniocentesis entre las semanas 13 y 37 de gestación y a 25 la biopsia de corion entre las semanas 8 y 13 de edad gestacional. Las indicaciones según el tipo de muestra analizada se presentan en la tabla 1.

\section{Resultados de las muestras de LA}

La mayoría de las muestras presentaron el aspecto y color considerados normales, con excepción de 7 muestras que aunque estaban sanguinolentas no se vieron afectadas en el desarrollo del cultivo. La cantidad de LA varió dependiendo de la edad gestacional y el promedio fue de $19.3 \mathrm{mls}$. Todas las muestras estuvieron en condiciones de asepcia. El tiempo invertido en alcanzar un número adecuado de células (mínimo 7 colonias con buen índice mitótico) fue de 16.8 días en promedio para los cultivos en frasco y 11 días en promedio para los cultivos in situ. Estos cultivos tuvieron un buen número de metafases (aproximadamente 5 por campo) y buena calidad de bandas. El análisis citogenético fue posible en 85 casos $(97.7 \%)$ Tablas 2 y 3. En ninguna de las muestras con resultado citogenético masculino fue detectada contaminación celular materna (CCM). Se presentó mosaicismo en 1 caso (1.17\%). Este mosaico resultó ser un falso positivo y fue correctamente diagnosticado. Los resultados citogenéticos se muestran en la tabla 3 y figura 1.

\section{Resultados de las muestras de VC}

En la población estudiada hubo 2 muestras inadecuadas, 1 que sólo tenía decidua y 1 con cantidad insuficiente de VC (menos de $5 \mathrm{mgs}$.). En ellas hubo necesidad de analizar el LA para lograr el diagnóstico citogenético. Hubo otras 3 muestras con poca cantidad de vellosidades (entre 5 y $7 \mathrm{mgs}$.) que presentaron dificultad en el análisis, pero se pudo realizar el diagnóstico. Las demás muestras (80\%) tenían $10 \mathrm{mgs}$. o más de VC y estaban en buenas condiciones de vitalidad. Hubo una de estas
Tabla 1

\begin{tabular}{|c|c|c|c|}
\hline \multirow{2}{*}{ Indicaciones } & \multicolumn{2}{|c|}{ Tipo de procedimiento } & \multirow{2}{*}{ Total } \\
\hline & $\begin{array}{l}\text { Amnio- } \\
\text { centesis }\end{array}$ & $\begin{array}{c}\text { Biopsia } \\
\text { Vellosidades } \\
\text { coriales }\end{array}$ & \\
\hline $\begin{array}{l}\text { Edad materna } \\
\text { avanzada }\end{array}$ & $\begin{array}{c}50 \\
(57.5 \%)\end{array}$ & $\begin{array}{c}20 \\
(80 \%)\end{array}$ & $\begin{array}{c}70 \\
(62.5 \%)\end{array}$ \\
\hline Antecedentes & $\begin{array}{c}17 \\
(19.5 \%)\end{array}$ & $\begin{array}{c}5 \\
(20 \%)\end{array}$ & $\begin{array}{c}22 \\
(19.64 \%)\end{array}$ \\
\hline $\begin{array}{l}\text { Obstétricas } \\
\text { o fetales }\end{array}$ & $\begin{array}{c}20 \\
(23 \%)\end{array}$ & $\begin{array}{c}0 \\
(17.85 \%)\end{array}$ & 20 \\
\hline Total & $\begin{array}{c}87 \\
(100 \%)\end{array}$ & $\begin{array}{c}25 \\
(100 \%)\end{array}$ & $\begin{array}{c}112 \\
(100 \%)\end{array}$ \\
\hline
\end{tabular}

Indicaciones de las 112 pacientes según el tipo de procedimiento utilizado.

Tabla 2

\begin{tabular}{|c|c|c|}
\hline \multirow{2}{*}{ Resultado_ } & \multicolumn{2}{|c|}{ Tipo de muestra } \\
\hline & $\begin{array}{l}\text { Líquido } \\
\text { Amniótico }\end{array}$ & $\begin{array}{l}\text { Vellosidades } \\
\text { Coriales }\end{array}$ \\
\hline $\begin{array}{l}\text { Cariotipo } \\
\text { normal }\end{array}$ & $80(91.9 \%)$ & $19(76 \%)$ \\
\hline $\begin{array}{l}\text { Alteración } \\
\text { citogenética }\end{array}$ & $4(4.6 \%)$ & $2(8 \%)$ \\
\hline $\begin{array}{l}\text { Falsos } \\
\text { positivos }\end{array}$ & $1(1.15 \%)$ & $1(4 \%)$ \\
\hline $\begin{array}{l}\text { Falla por muestra } \\
\text { inadecuada }\end{array}$ & & $2(8 \%)$ \\
\hline $\begin{array}{l}\text { Falla en el } \\
\text { procesamiento } \\
\text { laboratorio }\end{array}$ & $2(2.3 \%)$ & $1(4 \%)$ \\
\hline Total & $87(100 \%)$ & $25(100 \%)$ \\
\hline
\end{tabular}

Resultados citogenéticos de las 112 pacientes de acuerdo al tipo de muestra procesada. 
Tabla 3

\begin{tabular}{|ccc|}
\hline & \multicolumn{2}{c|}{ Tipo de muestra } \\
\cline { 2 - 3 } Ciagnóstico & & \\
\cline { 2 - 3 } & $\begin{array}{c}\text { Líquido } \\
\text { Amniótico }\end{array}$ & $\begin{array}{c}\text { Vellosidades } \\
\text { Coriales }\end{array}$ \\
\hline $46, \mathrm{XY}$ & $47(55.3 \%)$ & $7(31.81 \%)$ \\
$46, \mathrm{XX}$ & $33(38.82 \%)$ & $12(54.54 \%)$ \\
$46, \mathrm{XYnf}$ & $1(1.17 \%)$ & \\
$47, \mathrm{XX},+21$ & $1(1.17 \%)$ & $1(4.54 \%)$ \\
$47, \mathrm{XX},+18$ & $2(2.35 \%)$ & \\
$45, \mathrm{XY}, \mathrm{t}(14 ; 21)$ & & $1(4.54 \%)$ \\
Confinados: & & \\
(falsos positivos) & & $1(4.54 \%)$ \\
mos 46,XY/92,XXYY & $1(1.17 \%)$ & $22(100 \%)$ \\
$92, \mathrm{XXYY}$ & & \\
\hline Total & $85(100 \%)$ & \\
\hline
\end{tabular}

Resultados de los cariotipos de las 106 muestras en las que se obtuvo el diagnóstico citogenético. últimas muestras en la que fue necesario recurrir a la amniocentesis por un error de laboratorio en el procesamiento. De las muestras adecuadas se obtuvieron en promedio 3 láminas por paciente, 10 metafases estudiables por lámina en cultivos de 48 horas y 7 metafases estudiables por lámina en cultivos de 24 horas, el bandeo estuvo adecuado en la mitad de estas metafases. Los resultados se informaron entre 8 y 15 días después de la toma de la muestra. Se obtuvieron resultados citogenéticos en 22 casos o sea en el $95.6 \%$ de las muestras tomadas adecuadamente, tablas 2 y 3 . No se detectó CCM en los casos con resultado citogenético masculino. Se presentó un caso de un falso positivo, el cual correspondió a un 4.5\%. Los resultados citogenéticos se muestran en la tabla 3 y figura 1.

\section{Discusión}

La menor cantidad de muestras de $\mathrm{VC}$ con respecto a las de LA ( 25 versus 87 ), se debe a que en nuestro medio no se ha promovido suficientemente la biopsia de corion. Son pocos los gineco-obstetras capacitados para practicarla y son muchos los que manejan conceptos erróneos e imparciales de la literatura al respecto. Sin embargo, lentamente hemos ido ganando experiencia en este tipo de.procedimiento tanto a nivel obstétrico como citogenético.

La anterior situación contrasta con la amniocentesis la cual se popularizó desde hace muchos años, y es realizada en varias ciudades del país por un número de

Figura 1

ANORMALIDADES CITOGENETICAS ENCONTRADAS EN LAS MUESTRAS ESTUDIADAS EN LIQUIDO AMNIOTICO (A) Y VELLOSIDADES CORIONICAS (B).

Cromosomas $\mathrm{X}$ y $\mathrm{Y}$ coloreados con mostaza de quinacrina, los demás con colorante giemsa, $1250 \mathrm{x}$

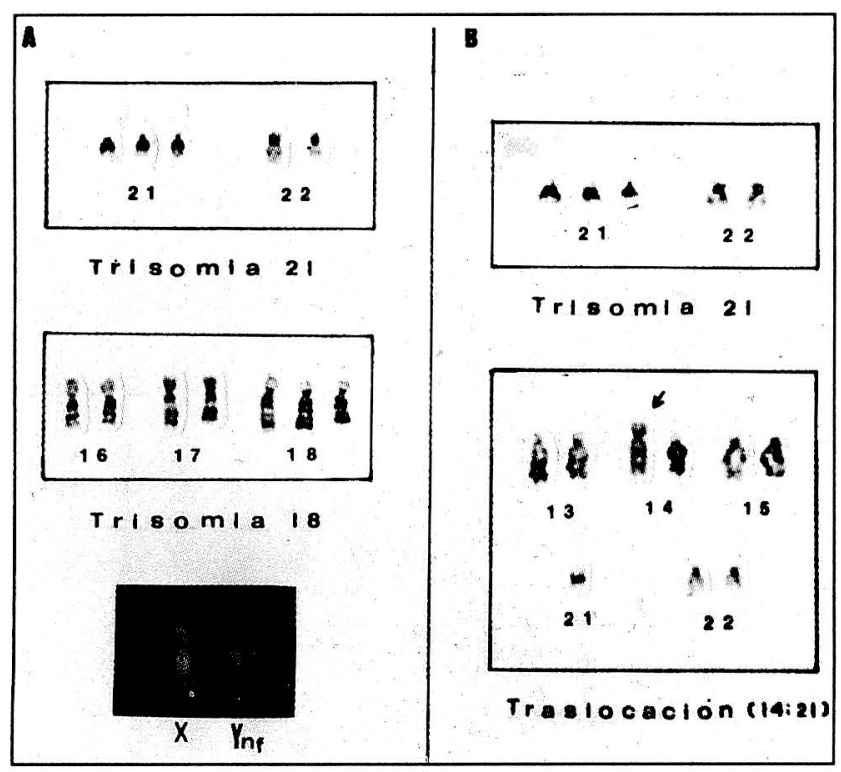


gineco-obstetras cada vez mayor, algunos de los cuales la practican incluso desde la semana 12 de gestación. Por esta razón la cantidad y calidad de las muestras de LA fue en su mayoría muy buena y aunque hubo algunas sanguinolentas esta condición no afectó el crecimiento celular.

La obtención de la biopsia de corion en condiciones adecuadas es más exigente. En este estudio se encontraron 2 muestras inadecuadas ( $8 \%$ ), aunque este es un porcentaje alto, la tendencia ha ido disminuyendo a medida que se ha ganado experiencia.

El tiempo promedio para la obtención de un buen número de células de LA en división activa fue de 16.8 días, mientras que en VC, los cultivos tanto de 24 como 48 horas fueron suficientes para encontrar metafases adecuadas. Si a esto se suma el hecho de que la muestra de VC es tomada varias semanas antes que la de LA, aún considerando la amniocentesis temprana, se hace evidente la mayor ventaja que posee el diagnóstico prenatal citogenético en VC, que es precisamente la obtención de un resultado mucho más rápido.

En promedio el informe del LA se estuvo entregando 20 días después de la toma de la muestra y el de VC 10 días después de la biopsia, lo que significa que con VC el resultado se conoció alrededor de 3 semanas antes que con LA.

Sin embargo con las VC hay muchas más exigencias en cuanto al tiempo de dedicación, los cuidados y la experiencia necesaria para el estudio citogenético, que con los LA. Esto se debe a que tanto los cromosomas como las bandas obtenidas son de menor calidad y las células presentan mayor susceptibilidad al rompimiento después de que ha sido procesado del cultivo.

La obtención del diagnóstico citogenético fue alta tanto para las muestras de LA (97.6\%), como para las de VC $(95.6 \%)$, cercana a las reportadas en la literatura: $99.7 \%$ en LA (15) y $98.5 \%$ en VC (16). El resultado obtenido fue exitoso a pesar de los errores circunstanciales, más que todo técnicos, que se nos presentaron. En el LA no hubo crecimiento celular en las dos primeras muestras sembradas, debido a un problema intrínseco de la incubadora que fue debidamente corregido. En las VC hubo una muestra en la que no fue posible obtener el resultado citogenético debido a un error humano cometido durante el procesamiento durante la cosecha, y en dos muestras por insuficiencia de tejido.

Como era de esperarse, en este tipo de estudio se encontraron aberraciones cromosómicas tanto numéricas como estructurales. Los estudios realizados en muestras de LA tuvieron un $4,7 \%$ de anormalidades cromosómicas ( $75 \%$ numéricas y $25 \%$ estructurales), mientras que los realizados en VC mostraron un $9 \%$ de anormalidades $(50 \%$ de ambas alteraciones, numéricas y estructurales). En la literatura se reporta un $1.22 \%$ en LA (17) y $3.5 \%$ en VC (18). Nuestros hallazgos muestran frecuencias más elevadas debido probablemente al tamaño de la muestra, por lo que es importante ampliarla.
En el diagnóstico prenatal los dos problemas que requieren mayor cuidado al realizar la interpretación citogenética son: (1) el mosaicismo y (2) la CCM.

(1) El mosaico es la presencia de dos o más líneas celulares con diferente complemento cromosómico. Hay dos clases de mosaicismo, el verdadero, que es el que está presente en el feto, y el pseudomosaicismo que es un mosaico detectado en los cultivos celulares pero que no es representativo de la constitución cromosómica del feto. El pseudomosaico puede estar confinado a la placenta, ser producto de una mutación «in vitro» o ser un artefacto del cultivo $(19,20,21)$. Por consiguiente, para hacer correctamente el diagnóstico citogenético hay que saber distinguir entre estos dos tipos de mosaicismo.

En el análisis citogenético del LA el pseudomosaicismo puede ser reconocido cuando es encontrada una sola célula con cariotipo anormal, en ese caso se denomina mosaicismo de nivel I (22). En este estudio el mosaicismo de nivel I ocurrió en 11 cultivos en los que se encontraron células aneuploides, con pérdida de uno o más cromosomas, probablemente debido a rupturas celulares durante la manipulación del cultivo y un caso en el que se encontró una célula con una translocación balanceada 46, XY,t(4p;5q).

También se considera pseudomosaico cuando dos o más células de un frasco son encontradas con la misma anormalidad, mientras que las células de los demás clones y frascos son normales, en ese caso se denomina mosaicismo de nivel II (22). En este estudio no se encontró ningún mosaico de este tipo.

Cuando la alteración se encuentra en dos o más clones de los tres frascos, se habla de mosaicismo de nivel III (22), y esta es la situación más difícil de definir. En este estudio esa situación sólo se detectó en algunas células tetraploides, pero ya es conocido que este tipo de anormalidad se presenta con frecuencia (entre un $10 \%$ y un $83 \%$.) en cultivos de LA (23). Sin embargo, si la tetraploidía se encuentra en frascos de cultivo independientes, con una frecuencia significativa y consistente, se debe confirmar o descartar con otro cultivo, ya sea de LA o de sangre fetal, pues hay que tener en cuenta que en recién nacidos se han detectado mosaicismos involucrando tetraploidías $(24,25)$. En este estudio hubo un caso en el que se presentó este hallazgo citogenético y fue correctamente interpretado como un falso positivo, pues aunque la alteración fue encontrada en un $94 \%$ de las células de los tres frascos de cultivo (mosaicismo de nivel III), y aunque la paciente no aceptó la toma de otra muestra, se le hizo una exhaustiva evaluación ecográfica, y no se detectó ninguna de las malformaciones que estarían afectando a un feto con este tipo de mosaicismo.

De acuerdo con lo planteado anteriormente, todos los mosaicismos encontrados en los LA de este estudio fueron pseudomosaicos, sólo hubo un caso de nivel III, que fue el que presentó mayores dificultades de interpretación, y que es considerado como falso positivo, correspondiendo al 1,17\% de las muestras de LA. En la literatura 
este tipo de mosaicos se ha encontrado entre un $0.1 \%$ y un $0.3 \%(22,26,27,28)$.

En VC no se encontraron mosaicos pero el resultado de una de las muestras fue consistente con el planteamiento de un falso positivo. Esta situación puede surgir durante los primeros estados embrionarios, resultando en líneas celulares restringidas a algunos tejidos: si sólo están involucrados tejidos extra-embrionarios se conduciría a un falso positivo y si están restringidas al embrión, la línea celular no se detectaría en las VC y sería detectado como un falso negativo. En un caso de este estudio, aunque sólo se alcanzaron a estudiar 4 metafases, todas ellas presentaban tetraplodía. Al hacer la amniocentesis se encontró un cariotipo femenino completamente normal; también fue normal la evaluación clínica realizada a la niña al nacer. Por consiguiente el falso positivo en este estudio se presentó en una de las VC estudiadas y correspondió a un $4.54 \%$, en la literatura los falsos positivos se han reportado del $2 \%(29)$.

(2) La CCM es otra de las causas de error en la interpretación de los resultados. En estudios de LA se reporta una CCM de alrededor del $0.49 \%$ (30), y se plantea que ocurre con mayor probabilidad cuando ha habido más de un intento en la toma de la muestra, cuando ha sido muy escasa o cuando se ha obtenido sanguinolenta. En nuestro estudio no se observó este fenómeno el cual sólo se puede sospechar en caso de los fetos masculinos.

En las muestras de VC tampoco se encontraron células femeninas en los fetos con complemento cromosómico masculino, así que la CCM nó se detectó. Este hecho está de acuerdo con la experiencia previa, en donde la divi- sión espontánea de las células de la decidua, es de muy rara ocurrencia en preparaciones cromosómicas directas o en cultivos cortos de VC (15).

Entre las alteraciones estructurales encontradas hubo una deleción de los brazos largos del cromosoma Y que fue detectada en una muestra de LA. El cromosoma Y humano presenta una gran variación de tamaño en la población en general (31). Este polimorfismo es debido a la heterocromatina constitutiva que se encuentra en la parte distal de los brazos largos, y muestra un intenso patrón de fluorescencia, cuando no se encuentra se denomina Ynf o cromosoma Y no fluorescente (figura 1). La longitud de la región eucromática de este cromosoma es constante y no puede perder ningún segmento, pues en los varones con esta deficiencia se presentaría algún grado de infertilidad $(32,33)$.

Cuando hay una pérdida total de la región heterocromática, sin alteración de la zona eucromática, como en este caso, no se presenta infertilidad, como se demostró por la presencia del mismo cromosoma en el padre del feto en desarrollo. La deleción detectada en el feto y en el padre (del Yq12) ha sido descrita en varias ocasiones, sin la presencia de alteraciones clínicas ni fenotípicas $(34,35,36$.

\section{Agradecimientos}

A los doctores Jorge Orjuela y Eduardo Acosta, por su colaboración en el trabajo mediante la toma de muestras de biopsia de corion.

A Alfonso Garzón por su ayuda en el trabajo de fotografía.

\section{BIBLIOGRAFIA}

1. Steele MW., Breg WR. 1966. Chromosome analysis of human amniotic fluid cells. Lancet I:383.

2. Cornel M.C. Variation in prenatal cytogenetic diagnosis: policies in 13 european countries, 1989-1991. Pren Diag 1994; 14: 337-344.

3. American Academy of Pediatrics. Prenatal genetic diagnosis for pediatricians. Pediat 1994; 93:1010-1015.

4. Cha'ban F.K., Vans Plunder P., Los F.J., Wladimiroff J.W. Fetal outcome in nuchal translucency with emphasis on normal fetal Kariotype. Pren Diag 1996; 16:537-541.

5. Wald N.J., Densem J.W., George L., Muttukrishna S., Knight P.G. Prenatal screening for Down s syndrome using inhibin-A as a serum marker. Pren Diag 1996; 16: 143-153.

6. Chandley AC. 1981. The origin of chromosomal aberration in man and their potential for survival and reproduction in the adult human population. Ann Genet 24:5.

7. Jacobs PA, Browne C, Gregson N et al. 1992. Estimates of the frecuency of chromosome abnormalities detectable in unselected newborn using moderate levels of banding. J Med Genet 29:103.

8. Thompson MW, McInnes RR, Willard HF. Genetics in Medicine. 5th ed., Philadelphia: WB Saunders Company; 1991; 224.

9. Milunsky A. Genetic disorders and the fetus. New York: Plenun Press; 1986; 125.

10. Hsu LYF, Perlis FJ. United States Survey on chromosome mosaicism and pseudomosaicism in prenatal diagnosis. Prenat Diagn 1984; 4:97.
11. Gregson NM, Seabright M. Hadling chorionic villi for direct chromosomes studies. Lancet II: 1983; 1491.

12. Simoni G, Rossella F. First trimester fetal karyotyping using chorionic villi: technical development and diagnostic application. Experientia 1986; 42:1097.

13. Simoni G, Brambati B, Danesino C et al. Efficient direct chromosome analysis and enzime determinations from chorionic villi samples in the first trimester of pregnancy. Hum Genet 1983; 63:349.

14. Saura R, Longy M, Horowitz J et al. Biopsie de trophoblaste amélioration de la technique chromosomique. A propos de 200 cas. J Genet Hum 1988; 36:115.

15. Smidth-Jensen S, Lind AM, Permin M et al. Citogenetic nalysis of 2928 CVS samples and 1075 amniocentesis from randomized studies. Prenat Diagn 1993; 13:723.

16. Wang, BT., Peng, W., Cheng, K., et al. Chorionic villi sampling: Laboratory experience with 4000 consecutive cases. Am J Med Gen 1994; 53: 307-316.

17. Hsieh FJ, Ko TH, Tseng LH et al. Prenatal cytogenetic diagnosis in amniocentesis. Taiwan-I-Hsueh-Hui-Tsa-Chih 1992; 91:276.

18. Ledbetter DH, Martin AO, Verlinsky Y et al. Cytogenetic results of chorionic villus sampling: high success rate and diagnostic accuracy in the United States collaborative study. Am J Obstet Gynecol 1990; $162: 495$.

19. Kalousek DK, Dill FJ, Pantzar T et al. Confined chorionic mosaicism in prenatal diagnosis. Hum Genet 1987; 77:163. 
20. Stetten, G., Meissner, HI. Unusual and rapid amniotic fluid cell growth. Am J Obstet Gynecol 1981; 140: 719.

21. Artan S., Basaran N., Hassan H., Ozalp S. et al. Confined placental mosaicism in term placentae: analysis of 125 cases. Pren Diag 1995; 15: 1135-1142.

22. Hsu, LYF., Perlis, FJ. United States survey on chromosome mosaicism and pseudomosaicism in prenatal diagnosis. Pren Diag 1984; 4: 97.

23. Milunsky, A. Genetic disorders and the fetus. New York: Plenum Press; 1986: 167.

24. Golbus MS, Bachman R, Wiltse S y col. Tetraploidy in a liveborn infant. J Med Genet 1976; 13: 329.

25. Quiroz E, Orozco A, Salamanca F. Diploid-tetraploid mosaicism in a malformed boy. Clin Genet 1985; 27: 183.

26. Bui, T.H., Iselius, L., Lindsten, J. European collaborative study on prenatal diagnosis: mosaicism, pseudomosaicism and single abnormal cell in amniotic fluid cell cultures. Prenat Diagn (Special Issue) 1984; 4: $145-162$.

27. Worton, R.G., Stern, R. A Canadian collaborative study of mosaicism in amniotic fluid cell cultures. Prenat Diagn (Special Issue) 1984; 4: 131-144.

28. Hsu L.Y.F., Kaffe S., Jenkins E.C. y col. Proposed guidelines for diagnosis of chromosome mosaicism in amniocytes based on data derived from chromosome mosaicism and pseudomosaicism studies. Prenat Diagn 1992; 12: 555-573.
29. Canadian Collaborative CVS-Amniocentesis Clinical Trial Group. Multicentre randomised clinical trial of chorion villus sampling and amniocentesis. Lancet i: 1989; 1:6.

30. Wilson, M.G., Lin, M.S., Fujimoto, A., y col. Chromosome mosaicism in 6000 amniosenteses. Am J Med Genet 1989; 32: 506-513.

31. Hsu, L.Y.F., Benn, PA., Tannenbaum, HL., Perlis, TE., Carlson, AD. Chromosomal polimorphisms of $1,9,16$ and $\mathrm{Y}$ in 4 major ethnic groups. Am J Med Genet 1987; 26: 96-101.

32. Yunis, E., García- Conti, FL., de Caballero OMT., Giraldo, A. Yq deletion, aspermia and short stature. Hum Genet 1977; 39: 117-122.

33. Hsu L.Y.F. Phenotype/Karyotype correlations of $Y$ chromosome aneuploidy with emphasis on structural aberrations in postnatally diagnosed cases. Am J Med Genet 1994; 53: 108-140.

34. Langmaid, H., Laurence, KM. Deletion of the long arms of the $Y$ chromosome with normal male development and intelligence. J Med Genet 1974; 11: 208-211.

35. Magenis, E., Donlon, T. Nonfluorescent Y chromosomes. Cytologic evidence of origin. Hum Genet 1982; 60:133-138.

36. Muller, U., Donlon, T., Schmid, M., et al. Deletion mapping of the testes determining locus with DNA probes in 46, XX males and in 46,XY and 46,X,dic(Y) females. Nucleic Acids Res 1986; 14: 64896505 . 